\title{
COMPARISON STUDY OF EXECUTIVE FUNCTIONS IN PARKINSON'S DISEASE AND DEGENERATIVE CEREBELLAR DISEASE'S PATIENTS
}

To the Editor - It was with great interest that I read the article by Abel et al. describing the comparative study of executive functions in two groups of patients - the first with Parkinson's disease (PD) and the second with pure degenerative cerebellar disease $(C D)^{1}$. Based on an assessment of various different neuropsychological tests, the authors conclude that the group with CD had a greater deficit of executive functions with a prefrontal dysfunction pattern. The results of the study are of great importance and are in keeping with current understanding of the role of the cerebellum in cognitive functions. This function of the cerebellum has been stressed in a number of recent studies, particularly those of Schmahmann et $\mathrm{al}^{2,3}$. The group of patients with PD seemed to me to be particularly suitable for the study (mild to moderate forms with Hoehn-Yahr <3), with well-defined diagnostic criteria (Parkinson's Disease Society Brain Bank), unlike the group of patients with $C D$. This latter group consisted of a series of 14 patients with different illnesses, namely, spinocerebellar ataxia (SCA) (three cases with a dominant autosomal pattern, which were negative for SCA types 1, 2, 3, 7 and 8 ; three family cases without any etiologic investigation; two cases of SCA type 2; and one case of SCA type 1) and five cases defined as primary acquired cerebellar atrophy. The cases defined as SCA actually represent a range of very heterogeneous diseases that affect different systems (for example, the pyramidal, basal ganglia and peripheral nervous systems) and cognitive functions in various ways ${ }^{4,5}$. Of the more commonly found SCA forms, only type 6 and occasionally type 10 present as pure cerebellar ataxia ${ }^{6}$. Likewise, the five patients classified as primary acquired cerebellar atrophy may represent other diseases, such as ILOCA and MSA type $C^{7}$. The group defined as $C D$ is thus very heterogeneous and should not, in my opinion, be classified under the heading pure degenerative cerebellar disease, as this renders comparative analysis with the homogeneous group of PD patients difficult.

\section{REFERENCES}

1. Abel CG, Stein GS, Pereyra S, et al. Estudio comparativo de las funciones ejecutivas entre pacientes com enfermedad de Parkinson y pacientes com enfermidad degenerativa cerebelosa. Arq Neuropsiquiatr 2006; 64:814-823.

2. Schmahmann JD. The cerebellum and cognition. International Review of Neurobiology, Volume 41. San Diego: Academic Press, 1997.

3. Schamahmann JD, Sherman JC. The cerebellar cognitive affective syndrome. Brain 1998;121:561-579.

4. Klockgether T, Ludtke R, Kramer B, et al. The natural history of degenerative ataxia: a retrospective study of 466 patients. Brain 1998;121: 589-600.

5. Manto MU. The wide spectrum of spinocerebellar ataxias (SCAs). Cerebellum 2005;4:2-6.

6. Teive HAG, Roa BB, Raskin S, et al. Clinical phenotype of Brazilian families with spinocerebellar ataxia 10. Neurology 2004;63:1509-1512.

7. Schols L, Szymanski S, Peters S, et al. Genetic background of apparently idiopathic sporadic cerebellar ataxia. Hum Genet 2000;107:132-137.

Hélio Teive, MD, PhD

Movement Disorders Unit

Neurology Service

Hospital de Clínicas, Federal University of Paraná

E-mail: hagteive@mps.com.br

The Author's Reply - I am very grateful with your comments and I agree with you that the growing genetic heterogeneity discovered during the last decade for many neurological disorders show us that a wide range of genetic mutations locus could produce syndromes clinically or pathologically very similar. However the reverse is also true, and a same genotype within members of a family could manifest itself as different phenotypes. Furthermore, some patients with sporadic ataxia filling the clinical criteria of late onset cerebellar atrophy (ILOCA)', actually have an inherited disease such as happen with one of our patients included in this sample without any familiar history reported, where genetic studies indicated a SCA2. Other studies have described SCA type 3 in patients with ILOCA ${ }^{2,3}$.

The fact is that currently the clinical criteria of diagnose of many degenerative neurological disorders are identified on the basis of their clinical syndrome pointing to the topography of the pathology, the temporal course pointing to the mechanism and the findings on structural neuroimaging, correlating usually the clinical syndrome with the 
topography of lesion rather tan the specific pathology producing the lesion.

From a practical point of view, one may wonder if it is useful that all patients with a progressive ataxic syndrome should be genetically screened for diseases without a current treatment, as long as the clinical changes are well document and guide decision making. That is specially important in my country where the genetic studies are limited by: the low economic incomes from most of our patients, reliability of the genetic center, very few centers could does the genetic study at the expense of higher costs and the commercial availability for many of the genetic causes of SCA. From the point of view of research studies it would be suitable to genetically screen all patients presenting with a history of degenerative cerebellar disorder, inherited or not.

Unfortunately, the heterogeneity of sample when using models of cerebellar degenerative disease, was a common problem with many of the previous clinical studies in the literature investigating the cognitive functions of the cerebellum. As you mention from one of your citations, in the article "The cerebellar cognitive affective syndrome" 20 patients with cerebellar pathology were selected without a control group, being the patient's characteristic thirteen patients with stroke, three with postinfectious cerebellitis, three with cerebellar cortical atrophy without mention about genetic studies or familiar history of ataxia, and one with a midline tumour resected. Grafman ${ }^{5}$ assessed the executive functions of a group of twelve patients with cerebellar atrophy, but heterogeneous in its clinical diagnose, being 9 with pure cerebellar cortical, two with OPCA and one with probable MSA. One year later Appollonio ${ }^{6}$ published an article comparing the performance in memory of eleven patients with cerebellar degeneration based on history and clinical and radiologic findings, ranging in age from 20 to 73 years. In the article, there is no specific mention about extracerebellar pathology or familiar history of the patients studied. However, there is no mention about familiar history of ataxia. More recently Leroi et al. ${ }^{7}$ compared the psychopathology between degenerative cerebellar disease and Huntington's disease. The cerebellar group was constituted by 31 subjects, 16 with sporadic disease and 15 with a familiar history. The group of inherited ataxia, although genetically well identified, was heterogeneous in its composition: 2 SCA1, 1 SCA2, 2 SCA6, 1 SCA 8 and 6 with a genetic etiology unknown, 2 SCA3. In the sporadic group of cerebellar disease were cases of MSA. Furthermore, as another limiting factor, $19 \%$ of the cerebellar patients met criteria for dementia.

Although we agree with you about the heterogeneity under the umbrella of cerebellar degenerative disorders, this was a limitation up to present and our goal was to compare the effects on cognition produced by the clinical dysfunction of Basal Ganglia and Cerebellum employing two models of subcortical degenerative disease: the degenerative ataxias, sporadic or inherited, with neurological symptoms and signs restricted to the cerebellar dysfunction based on the clinical history, the neurologic examination and the structural neuroimaging ${ }^{1}$ and Parkinson's disease. Even idiopathic Parkinson's disease when performing inmunohistochemistry with antibodies to $\alpha$-synuclein display a wider distribution of pathology than initially thought within cortical and many subcortical structures $^{8}$.

We are very grateful for your comments, it allows us to improve our research and specially for the motivation to read it and the effort to helps us. We try as much as we can to identify the genetic causes of a degenerative cerebellar disease when possible.

\section{REFERENCES}

1. Klockgether T, Schroth G, Diener H, Dichgans J. Idiopathic cerebellar ataxia of late onset: natural history and MRI morphology. J Neurol Neurosurg Psychiatry 1990;53:297-305.

2. Ranum L, Chung M, Banfi S, et al. Molecular and clinical correlations in spinocerebellar ataxia type 1 (SCA1): evidence for familiar onset in the age of onset. Am J Hum Genet 1994;55:244-252.

3. Silveira I, Lopez-Cendes I, Kish S, et al. Frequencies of spinocerebellar ataxia 1, dentatorubropallidoluysian atrophy and Machado Joseph disease mutations in a large group of spinocerebellar ataxia patients. Neurology 1996;46: 214-218.

4. Schmahmann J, Sherman J. The cerebellar cognitive affective syndrome. Brain 1998;121:561-575.

5. Grafman, et al. Cognitive planning deficit in patients with cerebellar atrophy. Grafman Neurology 1992;42:1493-1496.

6. Appolllonio IM, Grafman J, Schartz V, Massaquoi S, Hallet M. Memory in patients with cerebellar degeneration. Neurology 1993;43:1536-1544.

7. Leroi I, O Eran E, Marsch L, et al. Psychopathology in patients with degenerative cerebellar diseases: a comparison to Huntington's disease. Am J Psychiatry. 2002;159:1306-1314.

8. Forno LS. Neuropathology of Parkinson's disease. J Neuropathol Exp Neurol 1996;27:255-259.

Carlos Gustavo Abel, MD Cognitive and Movement Disorders Unit Department of Neurology Ramos Mejia's Hospital Buenos Aires, Argentina E-mail: dakar58@arnet.com.ar 